\title{
DESIGN OF ROBUST PI CONTROLLERS AND THEIR APPLICATION TO A NONLINEAR ELECTRONIC SYSTEM
}

\author{
Radek Matušů ${ }^{*}$ Katarína Vaneková ${ }^{* *}$ \\ Roman Prokop * - Monika Bakošová **
}

\begin{abstract}
The principal aim of the paper is to present a possible approach to the design of simple Proportional-Integral (PI) robust controllers and subsequently to demonstrate their applicability during control of a laboratory model with uncertain parameters through the Programmable Logic Controller (PLC) SIMATIC S7-300 by Siemens Company. The proposed and utilized synthesis consists of two steps. The former one is determination of controller parameters area, which ensures the robustly stable control loop and is based on computing/plotting the stability boundary locus while the latter one lies in the final choice of the controller itself relying on algebraic techniques. The basic theoretical parts are followed by laboratory experiments in which the 3rd order nonlinear electronic model has been successfully controlled in various working points.

K e y w ords: robust control, PI controllers, parametric uncertainty, PLC simatic, nonlinear electronic system
\end{abstract}

\section{INTRODUCTION}

The contemporary industrial practice clearly prefers the usage of simple PI or PID controllers with fixed parameters despite the existence of many advanced control technologies. PI/PID compensators are well known, widespread and easily applicable, and so the investigation of an effective method of their tuning is still very topical, especially if these algorithms should be able to cope with various uncertain conditions.

Among an array of existing methods, eg [1,3,19], a possible way of robust controller design for systems with parametric uncertainty comprises the computation of all robustly stabilizing controllers and then the choice of the final one on the basis of additional user requirements. For the determination of (robustly) stabilizing controllers the method using the stability boundary locus plotting in combination with the sixteen plant theorem, which is described in [16] and [17], can be used. Besides, an elegant tool for control design itself can be adopted from the algebraic approach $[7,11,20]$. This technique is based on general solutions of Diophantine equations in the ring of proper and Hurwitz stable rational functions $\left(R_{P S}\right)$. It assumes the utilization of the Youla-Kučera parameterization, and conditions of divisibility in the specific ring. Furthermore, the selected controller can be further tuned through the only scalar tuning parameter $m>0$.

The paper deals with the problem how to find a simple continuous-time PI controller which robustly stabilizes and appropriately controls an interval plant. The novelty of the proposed approach consists in combination of plotting the stability boundary locus and choosing the final controller via an algebraic methodology. Furthermore, the work is focused not only on theoretical aspects of the proposed control design but also on its practical application in laboratory conditions. Thus, an electronic laboratory model has been identified as a system with parametric uncertainty and it has been controlled in various operational points using the designed simple PI algorithms which have been realized with the assistance of PLC SIMATIC S7-300.

The paper is organized as follows. In Section 2, basic theoretical background for computation of all possible robustly stabilizing PI controllers is provided. The Section 3 then contains the fundamentals of the algebraic approach to control design in $R_{P S}$ and applied controller tuning rules. Next, the Section 4 briefly describes SIMATIC industrial automation systems used in the practical part. Furthermore, the real laboratory experiments themselves in the meaning of model description, identification, calculations of controllers and real control results are presented in the extensive Section 5. And finally, Section 6 offers some conclusion remarks.

\section{COMPUTATION OF (ROBUSTLY) STABILIZING PI CONTROLLERS}

This part is intended to provide the basic ideas related to computing the PI controllers which stabilize the closed loop with fixed controlled system and consequently to extend the applicability of this approach for interval plants.

\footnotetext{
* Department of Automation and Control Engineering, Faculty of Applied Informatics, Tomas Bata University in Zlín, Nad Stráněmi 4511, 76005 Zlín, Czech Republic, \{rmatusu; prokop\}@fai.utb.cz, ${ }^{* *}$ Department of Information Engineering and Process Control, Faculty of Chemical and Food Technology, Slovak University of Technology in Bratislava Radlinského 9, 81237 Bratislava, Slovakia, \{katarina.vanekova; monika.bakosova\}@stuba.sk
} 


\subsection{Controlled systems with fixed parameters}

A potential approach to calculation of stabilizing PI controllers based on plotting the stability boundary locus is proposed in [16] and [17]. The method supposes the classical closed-loop control system with controlled plant

$$
G(s)=\frac{B(s)}{A(s)}
$$

and PI controller:

$$
C(s)=k_{p}+\frac{k_{I}}{s}=\frac{k_{p}+k_{I}}{s} .
$$

Initially, one needs to use the substitution $s=j \omega$ in the plant (1) and then to decompose the numerator and denominator of this transfer function into their even and odd parts

$$
G(j \omega)=\frac{B_{E}\left(-\omega^{2}\right)+j \omega B_{O}\left(-\omega^{2}\right)}{A_{E}\left(-\omega^{2}\right)+j \omega A_{O}\left(-\omega^{2}\right)} .
$$

Thereafter, expressing the closed-loop characteristic polynomial and setting the real and imaginary parts to zero lead to the equations

$$
\begin{aligned}
& k_{p}=\frac{\omega^{2} A_{0}\left(-\omega^{2}\right) B_{0}\left(-\omega^{2}\right)+A_{E}\left(-\omega^{2}\right) B_{E}\left(-\omega^{2}\right)}{-\omega^{2} B_{0}^{2}\left(-\omega^{2}\right)-B_{E}^{2}\left(-\omega^{2}\right)} \\
& k_{I}=\omega^{2} \frac{A_{E}\left(-\omega^{2}\right) B_{0}\left(-\omega^{2}\right)-A_{0}\left(-\omega^{2}\right) B_{E}\left(-\omega^{2}\right)}{-\omega^{2} B_{0}^{2}\left(-\omega^{2}\right)-B_{E}^{2}\left(-\omega^{2}\right)}
\end{aligned}
$$

Simultaneous solution of these relations and plotting the obtained values into the $\left(k_{p}, k_{I}\right)$ plane result in the stability boundary locus splitting the $\left(k_{p}, k_{I}\right)$ plane into the stable and unstable regions. Determination of the stabilizing area (or areas) can be done via a test point within each region. However, the proper frequency gridding is a potential problem. That is why this technique can be embellished with the Nyquist plot based approach from [14]. In this refinement, the frequency axis can be separated into several intervals by the real values of $\omega$ which fulfil

$$
\operatorname{Im}[G(s)]=0
$$

The stability or instability of a closed loop cannot change inside of frequency intervals resulting from (5) but only on their boundaries. Thus, such intervals are then very helpful for selection of the proper frequency scale.

\subsection{Improvement of the method for interval systems}

So far, the area of stabilizing controller coefficients for a given plant with only fixed parameters has been analyzed. However, the papers $[16,17]$ have improved the stabilization also for interval plants using the simple idea of its combination with the sixteen plant theorem $[1,2,4]$.
In compliance with this principle, a first order controller robustly stabilizes an interval plant

$$
G(s, b, a)=\frac{B(s, b)}{A(s, a)}=\frac{\sum_{i=0}^{m}\left[b_{i}^{-}, b_{i}^{+}\right] s^{i}}{\sum_{i=0}^{n}\left[a_{i}^{-}, a_{i}^{+}\right] s^{i}}, m<n
$$

where $b_{i}^{-}, b_{i}^{+}, a_{i}^{-}, a_{i}^{+}$are lower and upper bounds for numerator and denominator parameters, respectively, if and only if it stabilizes its 16 Kharitonov plants, which are defined as

$$
G_{i_{1}, i_{2}}(s)=\frac{B_{i_{1}}(s)}{A_{i_{2}}(s)}
$$

where $i_{1}, i_{2} \in\{1,2,3,4\}$; and $B_{1}(s)$ to $B_{4}(s)$ and $A_{1}(s)$ to $A_{4}(s)$ are the Kharitonov polynomials for the numerator and denominator of the interval system (6), respectively.

Remind that the Kharitonov polynomials eg for an interval polynomial

$$
B(s, b)=\sum_{i=0}^{m}\left[b_{i}^{+} ; b_{i}^{-}\right] s^{i}
$$

can be constructed using the upper and lower bounds of interval parameters according to the rule [5]:

$$
\begin{aligned}
& B_{1}(s)=b_{0}^{-}+b_{1}^{-} s+b_{2}^{+} s^{2}+b_{3}^{+} s^{3}+\ldots \\
& B_{2}(s)=b_{0}^{+}+b_{1}^{+} s+b_{2}^{-} s^{2}+b_{3}^{-} s^{3}+\ldots \\
& B_{3}(s)=b_{0}^{+}+b_{1}^{-} s+b_{2}^{-} s^{2}+b_{3}^{+} s^{3}+\ldots \\
& B_{4}(s)=b_{0}^{-}+b_{1}^{+} s+b_{2}^{+} s^{2}+b_{3}^{-} s^{3}+\ldots
\end{aligned}
$$

In other words, the stabilization of an interval plant follows from the stabilization of all 16 fixed Kharitonov plants. So the final stability region for an interval plant is given by intersection of all 16 related partial regions plotted individually according to the methodology from Subsection 2.1.

\section{Algebraic DESIGN OF A CONTROLlER}

The technique from the previous section allows determining the (robustly) stabilizing combinations of proportional and integral gains in PI controller. However, the final choice of the compensator is still an open question. A possible algebraic control design method has been developed in [7] and [20]. It is based on general solutions of Diophantine equations in $R_{P S}$. Moreover, it supposes the utilization of the known Youla-Kučera parameterization, which allows generating infinite amount of possible stabilizing controllers, while the choice of the final one depends on the desired properties mathematically represented by conditions of divisibility in the specific ring. Anyway, the selected controller can be further tuned. One of advantages of this approach is that behaviour of regulators can be influenced by the only scalar tuning parameter $m>0$. 


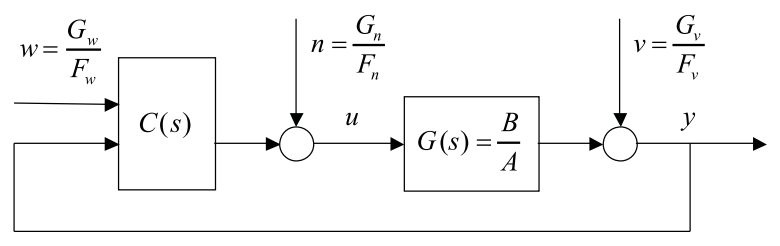

Fig. 1. General feedback control system

The synthesis assumes the description of linear systems in $R_{P S}$ as a ratio of two rational fractions

$$
\begin{gathered}
G(s)=\frac{b(s)}{a(s)}=\frac{b(s)(s+m)^{-n}}{a(s)(s+m)^{-n}}=\frac{B(s)}{A(s)}, \\
n=\max \{\operatorname{deg}(a), \operatorname{deg}(b)\}, m>0 .
\end{gathered}
$$

As it has been already mentioned, the scalar positive parameter $m>0$ will be later conveniently used as a "tuning knob".

A general feedback system is shown in Fig. 1. It represents the classical feedback one-degree-of-freedom (1DOF) control loop for $C(s)=\frac{Q(s)}{P(s)}$. Thus, the signal $u$ is generated according to the control law

$$
P(s) u=Q(s)[w-y]+P(s) n .
$$

In a two-degree-of-freedom (2DOF) control system, the controller $C(s)$ would consist of two transfer functions $\frac{Q(s)}{P(s)}$ and $\frac{R(s)}{P(s)}$. The control law is then governed by

$$
P(s) u=R(s) w-Q(s) y+P(s) n .
$$

The objective is to design controller transfer functions such that the feedback system is internally bounded-input bounded-output (BIBO) stable, the reference error tends asymptotically to zero and the disturbances $v$ and $n$ are asymptotically eliminated from the plant output. All transfer functions of the closed control system (Fig. 1) have a common denominator $A P+B Q$. One of the nice and convenient results of the algebraic philosophy is that this denominator should be a unit in the ring $R_{P S}$. In other words, the term $(A P+B Q)^{-1}$ resides in $R_{P S}$ and the feedback system is BIBO stable. If the elements $A$ and $B$ are coprime in $R_{P S}$ then all stabilizing controllers are given through an arbitrary solution $P_{0}, Q_{0}$ of Diophantine (Bézout) equation

$$
A P+B Q=1
$$

in a parametric form

$$
\frac{Q}{P}=\frac{Q_{0}-A T}{P_{0}+B T}
$$

where $T$ varies over $R_{P S}$ while satisfying $P_{0}+B T \neq$ 0 . This relation is known as (Bongiorno-)Youla-Kučera parameterization.
From the practical point of view, it is almost always desirable to ensure more than stability. The most frequent problem is that of reference tracking. Under assumption of zero disturbances $(n=v=0)$, algebraic analysis results in the fact that the tracking error $e$ tends to zero if

a) $F_{w}$ divides $A P$ for $1 \mathrm{DOF}$,

b) $F_{w}$ divides $(1-B R)$ for $2 \mathrm{DOF}$.

The last condition gives the second Diophantine equation in the form

$$
F_{w} Z+B R=1 .
$$

Details of this method can be found for example in [10$12]$.

From a set of developed results, this paper takes advantage of the $1 \mathrm{DOF}$ nominal tuning rules for the first order plant

$$
G(s)=\frac{b_{0}}{s+a_{0}} .
$$

Further, step-wise reference with $F_{w}=\frac{s}{s+m}$ and no disturbances are assumed. The Diophantine equation (13) takes the form

$$
\frac{s+a_{0}}{s+m} p_{0}+\frac{b_{0}}{s+m} q_{0}=1
$$

Multiplying by $(s+m)$, comparing coefficients and application of Youla-Kučera parameterization give the general stabilizing solution in the form

$$
P(s)=p_{0}+\frac{b_{0}}{s+m} T ; \quad Q(s)=q_{0}-\frac{s+a_{0}}{s+m} T
$$

where $q_{0}=\frac{m-a_{0}}{b_{0}} ; p_{0}=a$ and $T$ is free in $R_{P S}$. The asymptotic tracking for a stepwise reference $w$ will be given by divisibility of $F_{w}=\frac{s}{s+m}$ and $A P$. It is achieved for $T=t_{0}=-\frac{m}{b_{0}}$ so that $P(s)$ has a zero absolute coefficient in the numerator. Then inserting $t_{0}$ into (18) gives

$$
P(s)=\frac{s}{s+m} ; \quad Q(s)=\frac{\tilde{q}_{1} s+\tilde{q}_{0}}{s+m} .
$$

Thus, the final 1DOF feedback controller has the transfer function

$$
C(s)=\frac{Q(s)}{P(s)}=\frac{\tilde{q}_{1} s+\tilde{q}_{0}}{s}=\frac{k_{p} s+k_{I}}{s}
$$

which is a traditional PI control law governed by

$$
u(t)=k_{p}[w(t)-y(t)]+k_{I} \int[w(t)-y(t)] \mathrm{d} t
$$

and where

$$
k_{p}=\frac{2 m-a_{0}}{b_{0}} ; \quad k_{I}=\frac{m^{2}}{b_{0}} .
$$




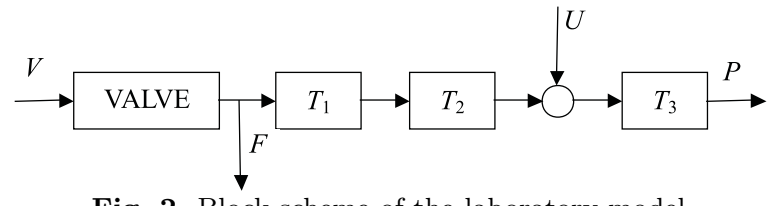

Fig. 2. Block scheme of the laboratory model

Table 1. Relation between $k$ and overshoot

\begin{tabular}{cc}
\hline Overshoot $(\%)$ & $k$ \\
\hline 0 & 1.00 \\
1 & 1.62 \\
2 & 1.87 \\
3 & 2.14 \\
4 & 2.44 \\
5 & 2.80 \\
6 & 3.25 \\
7 & 3.81 \\
8 & 4.58 \\
9 & 5.67 \\
10 & 7.38 \\
\hline
\end{tabular}

A possible way of additional controller tuning via $m>0$ represents the simple recommendation

$$
m=k a_{0}
$$

where the suitable coefficient $k$ can be chosen from the Tab. 1, which expresses the relation between $k$ and the size of first overshoot of the output (controlled) variable in percentage.

The derivation of presented nominal tuning results and some extending analyses are available $e g$ in [8].

\section{SIMATIC INDUSTRIAL AUTOMATION SYSTEMS}

For the purpose of experiments presented in the paper, not only theoretical background is necessary, but also the technical realization of the algorithms must be in the interest. The SIMATIC systems offer a complex solution for various tasks of industrial automation. From the control engineer point of view, the very attractive devices are included in the SIMATIC series of modular PLCs called SIMATIC S7.

\subsection{PLC SIMATIC S7-300}

Among the number of products, the controller SIMATIC S7-300 is a reasonable automation system for many process control applications, especially with the focus on manufacturing engineering, both in centralized and distributed configurations. The advantage is the ability to integrate a wide range of powerful CPUs with Industrial Ethernet/PROFINET interface, an array of available modules, integrated technological functions, or failsafe designs.

\subsection{SIMATIC S7 software equipment}

The programming of SIMATIC controllers, user's data accessing and, generally, the monitoring and control of real processes is performed through the programming (STEP7) and visualization (WinCC) software. The structure of the user's program is created by the organization block OB35 which represents the main program working in cycles with the period of $100 \mathrm{~ms}$. The OB35 then includes all needful function blocks, such as a continuous PID controller (FB41).

The set of several tasks must be done before programming the blocks $[6,13]$. It is necessary to create a project, configure a network, define input and output modules and define connections between them. Visualization of the project is realized in the Graphics Designer which is a part of WinCC system. Software tool WinCC gives possibility to define user's own visualization for controlled processes through the various manipulating elements, I/O fields, monitoring windows, etc. WinCC processes all important data from the program STEP7 while the connection between WinCC and STEP7 is linked by tags.

\section{REAL LABORATORY EXPERIMENTS}

The following section successively presents the practical application of the proposed control design method going from model description, continuing through identification experiments and calculations of two suitable controllers and finishing with the real control results.

\subsection{Model description}

The laboratory process is a nonlinear electronic model, which includes a 3rd order system with a variable time constant, adjustable from $5 \mathrm{~s}$ to $20 \mathrm{~s}$, and a model of nonlinear valve. The block scheme of the laboratory process is in Fig. 2, where signals are denoted as follows

$V$ - control signal for valve opening $(0-10 \mathrm{~V})$

$F$-signal representing the valve opening $(0-10 \mathrm{~V})$

$P$ - output of the process $(0-10 \mathrm{~V})$

$U$ - disturbance $(0-10 \mathrm{~V})$

\subsection{Identification}

The laboratory model was assumed as a black box and it was identified using step response experiments and Strejc method [15] in the form of a transfer function

$$
G(s)=\frac{K}{(T s+1)^{n}} e^{-D s}
$$

thus as a $n$-th order plant with multiple time constant $T$, gain $K$ and time-delay $D$. The step responses were measured in various working areas which led to the transfer function with parametric uncertainty

$G(s, K, T, D)=\frac{K}{(T s+1)^{2}} e^{-D s}=\frac{K}{T^{2} s^{2}+2 T s+1} e^{-D s}$ 
where $K, T$, and $D$ are supposed to vary within intervals obtained from minimum and maximum measured values [18]

$$
\begin{array}{ll}
K \in\left[K^{-}, K^{+}\right]=[0.37,5.186] & (-) \\
T \in\left[T^{-}, T^{+}\right]=[9.2909,11.0733] & (\mathrm{sec}) \\
D \in\left[D^{-}, D^{+}\right]=[0.966,2.181] & (\mathrm{sec})
\end{array}
$$

The denominator of model (25) has a single parameter polynomial structure of uncertainty and in the following considerations it will be overbounded by interval polynomial. Thanks to the fact, that the mutual dependence between two polynomial coefficients has been ignored, the potential robust stability tests will provide results with "only" stronger sufficient and not necessary and sufficient condition. However robust stability of the overbounding system would entail that also the original object is robustly stable.

So, the final overbounding interval system with timedelay is

$$
\begin{aligned}
& G_{0}(s, K, T, D)= \\
& \frac{\left[K^{-}, K^{+}\right]}{\left[\left(T^{-}\right)^{2},\left(T^{+}\right)^{2}\right] s^{2}+\left[2 T^{-}, 2 T^{+}\right] s+1} e^{-\left[D^{-}, D^{+}\right]} s .
\end{aligned}
$$

After insertion, the final transfer function appears

$G_{0}(s, K, T, D)=$

$$
\frac{[0.37,5.186]}{[86.3208,122.618] s^{2}+[18.5818,22.1466] s+1} e^{[0.966,2.181] s} \text {. }
$$

\subsection{Determination of robustly stabilizing PI controllers}

The computation of stabilizing PI controllers has been performed by method described in Section 2. However, first of all, it is necessary to put the transfer function into the form of rational function only, ie the time-delay term has to be approximated. For example, the first order Taylor expansion in denominator can be utilized. In other words, the object for future robust stability consideration has now moved from (25) or (27) to (29)

$$
\begin{aligned}
& G_{A}(s, K, T, D)= \\
& \frac{\left[K^{-}, K^{+}\right]}{\left[\left(T^{-}\right)^{2},\left(T^{+}\right)^{2}\right] s^{2}+\left[2 T^{-}, 2 T^{+}\right] s+1} \cdot \frac{1}{\left[D^{-}, D^{+}\right] s+1} \\
& =\left[K^{-}, K^{+}\right]\left\{\left[\left(T^{-}\right)^{2} D^{-},\left(T^{+}\right)^{2} D^{+}\right] s^{3}\right. \\
& +\left[2 T^{-} D^{-}+\left(T^{-}\right)^{2}, 2 T^{+} D^{+}+\left(T^{+}\right)^{2}\right] s^{2} \\
& \left.\quad+\left[D^{-}+2 T^{-}, D^{+}+2 T^{+}\right] s+1\right\}^{-1} \cdot
\end{aligned}
$$

The quantification leads to the approximated transfer function

$$
\begin{aligned}
& G_{A}(s, K, T, D)=[0.37,5.186]\left\{[83.3859,267.4298] s^{3}+\right. \\
& \left.[104.2708,170.9197] s^{2}+[19.5478,24.3276] s+1\right\}^{-1} \cdot
\end{aligned}
$$

The first of its 16 Kharitonov plants can be easily determined according to (7)

$$
G_{1,1}(s)=\frac{0.37}{267.4298 s^{3}+170.9197 s^{2}+19.5478 s+1} .
$$

Afterwards, the relations (4) have been expressed

$$
\begin{aligned}
& k_{p}=461.9451 \omega^{2}-2.7027 \\
& k_{I}=-722.7832 \omega^{4}+52.8319 \omega^{2}
\end{aligned}
$$

and then the suitable frequency interval for plotting the stability boundary locus can be computed using (5)

$$
\omega \in(0 ; 0.2704) \text {. }
$$

Simultaneous solving of the equations (32) and plotting the results into the $\left(k_{p}, k_{I}\right)$ plane leads to a stability region.

An analogical procedure must be repeated generally for all 16 Kharitonov plants. However, in this specific case, only the 8 plants are sufficient, because the numerator of (30) has only the gain with two extreme values and so it is not necessary to construct all 4 Kharitonov polynomials for such numerator.

Anyway, the stability regions for all 8 (16) Kharitonov plants are shown in Fig. 3 (interior areas).

The zoomed intersection of all stability regions from Fig. 3 determines the final area of robustly stabilizing PI controller coefficients for the original interval system (30) - see Fig. 4.

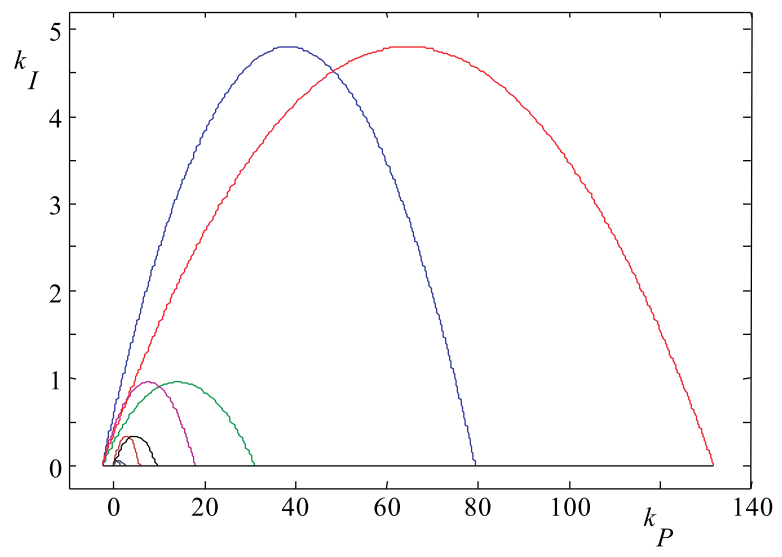

Fig. 3. Stability areas for 8, (16) - Kharitonov plants

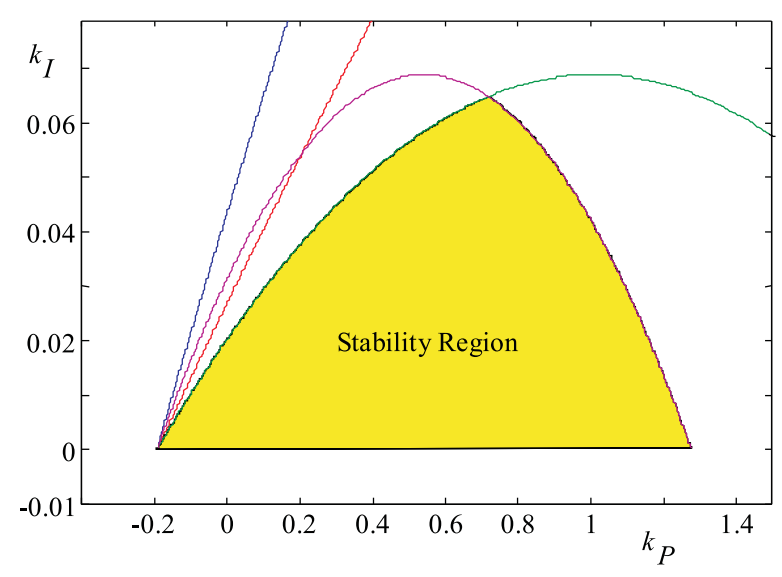

Fig. 4. Stability area for the interval system, (30) 


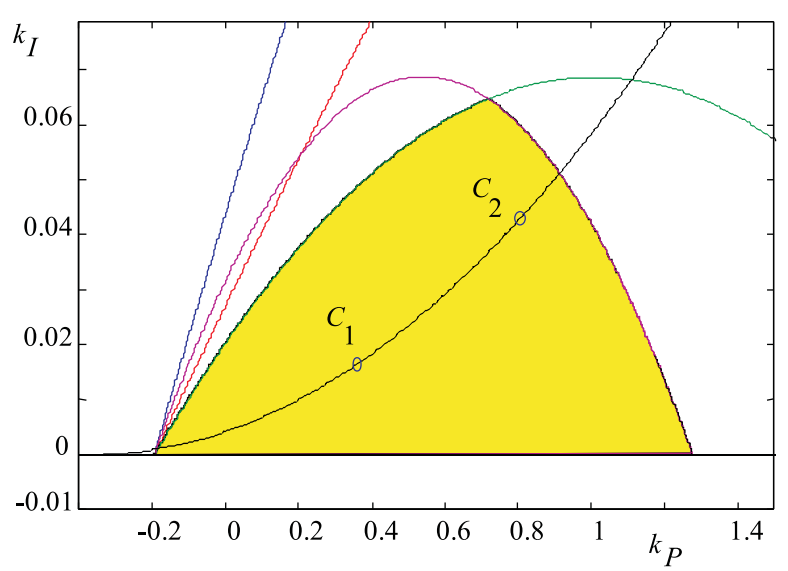

Fig. 5. Position of controllers, (36) - in stability region

\subsection{Controller choice}

Once the boundaries of robustly stabilizing PI coefficients are obtained, quite natural question emerges. How to find the practically suitable controller from this set?
Here, the algebraic approach from the Section 3 has been applied.

For the sake of appropriate order of the final controller (first order PI type), the controlled system must be described in the form of first order plant. The simple approximation of (29) leads to

$$
\begin{array}{r}
G_{A A}(s, K, D, T)=\frac{\left[K^{-}, K^{+}\right]}{\left[D^{-}+2 T^{-}, D^{+}+2 T^{+}\right] s+1} \\
=\frac{[0.37,5.186]}{[19.5478,24.3276] s+1}
\end{array}
$$

The average values of interval parameters then give the nominal plant (for control design)

$$
G_{N}(s)=\frac{2.778}{21.9377 s+1}=\frac{0.1266}{s+0.04558} .
$$

And now, the requirement of $0 \%$ and $1 \%$ first overshoot in controlled variable for the case of nominal system has been assumed. Thus, the utilization of appropriate parameters $k$ from Tab. 1 and, moreover, of the equations
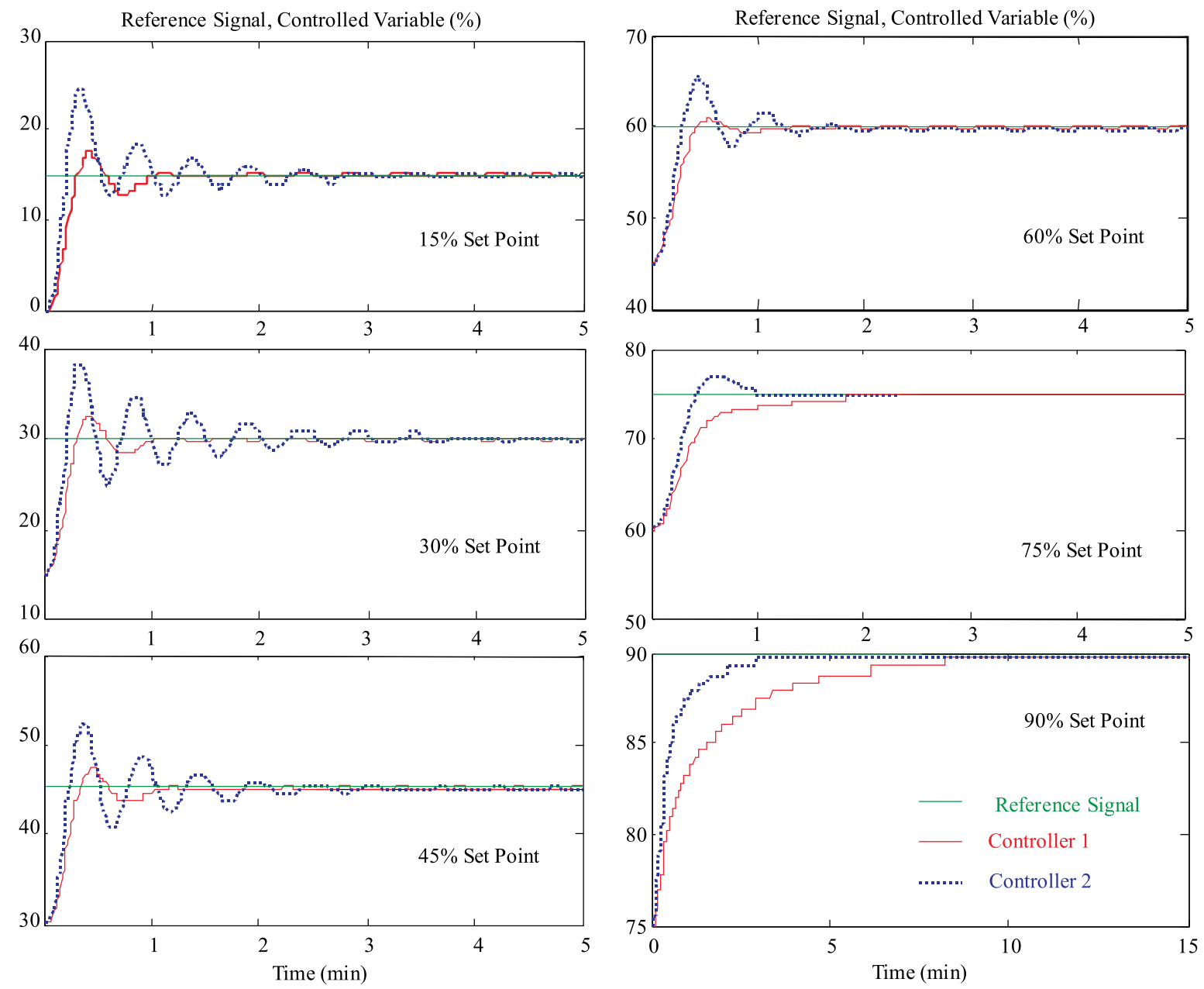

Fig. 6. Real control behaviour for various set points 
(23) and (22) result in the controller transfer functions

$$
\begin{aligned}
& 0 \% \Rightarrow m=0.04558 \Rightarrow C_{1}(s)=\frac{0.36 s+0.0164}{s} \\
& 1 \% \Rightarrow m=0.07384 \Rightarrow C_{2}(s)=\frac{0.8065 s+0.0431}{s}
\end{aligned}
$$

The position of these controllers in the stability region from Fig. 4 is depicted in Fig. 5. They lie on the curve which hypothetically connects the controllers tuned by various parameters $m$.

\subsection{Real control experiments}

Six control experiments have been performed for various reference points using the derived controllers and described hardware/software tools. The results are visualised in Fig. 6.

As expected, the prescribed overshoots were not possible to observe in real measurements (they holds true just for the nominal system), because of the plant nonlinearity. As can be seen, the "less aggressive" controller $C_{1}$ is very good mainly in middle levels of set points, however it has quite long settling time in higher areas. At the other side, the controller $C_{2}$ is much "faster" here, but it is oscillating in the lower set points. All in all, both controllers are able to control the process robustly stable with acceptable behaviour and the choice of the controller would depend on the main operational condition and user consideration.

\section{CONCLUSIONS}

Proportional-Integral controller design based on combination of plotting the stability boundary locus and the algebraic methodology has been proposed and successfully tested in laboratory conditions. The developed synthesis represents relatively easy and effective way of tuning of controllers for interval plants. On the other hand, coincident robust stability and nominal performance can not be assured in advance but they have to be verified during the design process which can be seen as a drawback of the approach. For the purpose of this paper, the designed algorithms have been technically realized during control of nonlinear 3rd order electronic model assumed as system with parametric uncertainty using control loop on the basis of PLC SIMATIC S7-300.

\section{Acknowledgments}

The authors would like to acknowledge the financial support from the Ministry of Education, Youth and Sports of the Czech Republic under Research Plan No. MSM 7088352102 and the Scientific Grant Agency of the Slovak Republic under Grants No. 1/0537/10 and $1 / 0071 / 09$. A shorter version of this paper has been presented at the 6th IFAC Symposium on Robust Control Design [9].

\section{REFERENCES}

[1] BARMISH, B. R. : New Tools for Robustness of Linear Systems, Macmillan, New York, USA, 1994.

[2] BARMiSH, B. R.-HOLLOT, C. V.-KRAUS, F. J.-TEMPO, R.: Extreme Point Results for Robust Stabilization of Interval Plants with First Order Compensators, IEEE Transactions on Automatic Control 37 (1992), 707-714.

[3] Bhattacharyya, S. P. 'CHAPELlat, H.-KEEL, L. H. : Robust Control: The Parametric Approach, Prentice Hall, Englewood Cliffs, New Jersey, USA, 1995.

[4] HO, M. T.-DATTA, A.-BHATTACHARYYA, S. P. : Design of P, PI and PID Controllers for Interval Plants, In: Proceedings of American Control Conference, Philadelphia, USA, 1998.

[5] KHARITONOV, V. L.: Differentsial'nye Uravneniya 14 (1978), 2086-2088.

[6] KOŽKA, S̆.-KVASNICA, M. : Programovanie PLC SIMATIC (Programming of PLC SIMATIC), Slovak University of Technology in Bratislava, Slovakia, 2001. (In Slovak)

[7] KUČERA, V.: Diophantine Equations in Control - A survey, Automatica 29 No. 6 (1993), 1361-1375.

[8] MATUŠŮ, R.-PROKOP, R. : Single-Parameter Tuning of PI Controllers: from Theory to Practice, In: Proceedings of the 17th IFAC World Congress, Seoul, Korea, 2008.

[9] MATUŠŮ, R.-VANEKOVÁ, K.-PROKOP, R.-BAKOŠOVÁ, M. : Robust Proportional-Integral Control of a Laboratory Model using Programmable Logic Controller SIMATIC S7-300, In: Proceedings of the 6th IFAC Symposium on Robust Control Design, Haifa, Israel, 2009.

10] PROKOP, R.-MÉSZÁROS, A.: Design of Robust Controllers for SISO Time Delay Systems, J. Electrical Engineering 47 No. 11-12 (1996), 287-294.

[11] PROKOP, R.-CORRIOU, J. P. : Design and Analysis of Simple Robust Controllers, Internat. J. Control 66 No. 6 (1997), 905-921.

[12] PROKOP, R.-HUSTÁK, P.-PROKOPOVÁ, Z. : Simple Robust Controllers: Design, Tuning and Analysis, In: Proceedings of the 15th IFAC World Congress, Barcelona, Spain, 2002.

[13] Siemens User Manual Standard Software for S7-300 and S7-400 PID Control. Siemens Automation Group, (1996).

[14] SÖYLEMEZ, M. T.-MUNRO, N.-BAKI, H. : Fast Calculation of Stabilizing PID Controllers, Automatica 39 No. 1 (2003), $121-126$

15] STREJC, V.: Regelungstechnik 7 (1959), 124-128, Approximation aperiodischer Übertragungscharakteristiken (Approximation of Aeriodic Step Responses). (In German)

[16] TAN, N.-KAYA, I. : Computation of stabilizing PI Controllers for Interval Systems, In: Proceedings of the 11th Mediterranean Conference on Control and Automation, Rhodes, Greece, 2003.

[17] TAN, N.-KAYA, I.-YerogLU, C.-ATHERTON, D. P.: Computation of Stabilizing PI and PID Controllers using the Stability Boundary Locus, Energy Conversion and Management 47 No. 18-19 (2006), 3045-3058.

[18] VANEKOVÁ, K.-BAKOŠOVÁ, M.-ZÁVACKÁ, J.: Robust PI Control of a Laboratory Process using Control System Simatic, In: Proceedings of 16th International Conference on Process Control, Štrbské Pleso, Slovakia, 2007.

[19] VESELÝ, V.-ROSINOVÁ, D.-KUČERA, V. : Robust Controller Design for Polytopic Systems via Robust Elimination Lemma, J. Electrical Engineering 60 No. 1 (2009), 34-38.

[20] VIDYASAGAR, M.: Control System Synthesis: a Factorization Approach, MIT Press, Cambridge, M.A., USA, 1985.

Received 6 February 2009 
Radek Matušủ (Ing, PhD), born in 1978, is a Researcher at the Faculty of Applied Informatics of Tomas Bata University in Zlín, Czech Republic. He graduated from the Faculty of Technology of the same university with an MSc in Automation and Control Engineering in 2002 and he received a $\mathrm{PhD}$ in Technical Cybernetics from the Faculty of Applied Informatics in 2007. He worked as a Lecturer from 2004 to 2006. The main fields of his professional interest include robust systems and application of algebraic methods to control design.

Katarína Vaneková (Ing), born in 1979, graduated from the Slovak Technical University in Bratislava, Faculty of Chemical and Food Technology. At present she is a PhD student at the Institute of Information Engineering, Automation and Mathematics, FCFT STU in Bratislava. Her field of research includes robust control of the chemical processes with uncertainties and the industrial control systems.

Roman Prokop (Prof, Ing, CSc), born in 1952, is a Vicedean and Full Professor at the Faculty of Applied Informatics of the same university. He graduated from the Czech Technical University in Prague in 1976 and received a $\mathrm{PhD}$ from the Slovak University of Technology in Bratislava in 1983. He was an Associate Professor from 1996 and Full Professor from 2004. He aims his pedagogical and research work to automatic control theory, algebraic methods in control design and optimisation. The main interests of the latest period are uncertain and robust systems, autotuning of controllers and time-delay systems.

Monika Bakošová (Doc, Ing, PhD) was born in 1958. She graduated from the Faculty of Chemical and Food Technology, Slovak University of Technology in Bratislava in 1982. She received her $\mathrm{PhD}$ degree in 1987 on hierarchical control of large-scale systems. In 1999 she was appointed Associate Professor at the FCFT. Since 1986 she has been with the STU in Bratislava and currently works at the Institute of Information Engineering, Automation and Mathematics, FCFT STU in Bratislava. Her research interests are focused on modelling, simulation and control of processes in chemical technology with special emphasis for the problems of robust stabilization and robust control.

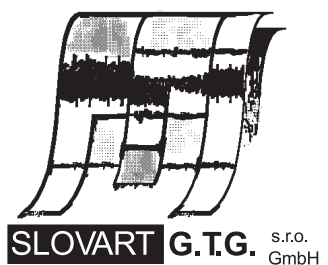

EXPORT - IMPORT
EXPORT - IMPORT

of periodicals and of non-periodically printed matters, books and CD-ROMs

Krupinská 4 PO BOX 152, 85299 Bratislava 5, Slovakia tel: ++421263839 472-3, fax: ++421263839485 info@slovart-gtg.sk; http://www.slovart-gtg.sk

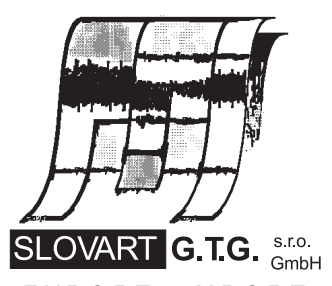

EXPORT - IMPORT 